Jurnal Matematika UNAND

Vol. VIII No. 2 Hal. $7-14$

Edisi Agustus 2019

ISSN : 2303-291X

(C)Jurusan Matematika FMIPA UNAND

\title{
SIFAT-SIFAT MATRIKS ORTOGONAL DAN TRANSFORMASI ORTOGONAL
}

\author{
ADIB ABDUL MAJID, YANITA, NOVA NOLIZA BAKAR \\ Program Studi S1 Matematika, \\ Fakultas Matematika dan Ilmu Pengetahuan Alam, Universitas Andalas, \\ Kampus UNAND Limau Manis Padang, Indonesia. \\ email : majidadibabdul@gmail.com
}

Diterima 22 Juni 2019 Direvisi 6 Juli 2019 Dipublikasikan 4 Agustus 2019

\begin{abstract}
Abstrak. Matriks ortogonal merupakan salah satu bentuk khusus dari jenis-jenis matriks. Suatu matriks dikatakan ortogonal ketika vektor-vektor nya mempunyai hasil kali titik sama dengan 0. Pada makalah ini akan dibuktikan sifat-sifat matriks ortogonal dan transformasi ortogonal, dan bagaimana keduanya terkait. Karena transformasi linier dapat diwakilkan oleh matriks, oleh karena itu jika suatu transformasi liniernya ortogonal, maka suatu matriksnya juga ortogonal.
\end{abstract}

Kata Kunci: Matriks ortogonal, Transfromasi Ortogonal

\section{Pendahuluan}

Matriks adalah susunan bilangan-bilangan dalam bentuk persegi panjang yang disusun berdasarkan baris dan kolom serta diapit oleh tanda kurung. Bilanganbilangan dalam persegi panjang tersebut dinamakan entri matriks. makalah ini mengambil salah satu jenis matriks khusus, yaitu matriks ortogonal, dimana sebuah matriks bujursangkar A yang memiliki sifat

$$
A^{-1}=A^{T} .
$$

Terdapat beberapa sifat yang diakibatkan karena kekhususannya ini, Penulis mengangkat kajian matriks ortogonal. Selain itu, karena transformasi linier dapat diwakilkan oleh matriks, oleh karena itu jika suatu transformasi liniernya ortogonal, maka suatu matriksnya juga ortogonal.

Oleh karena matriks dan transformasi linier mempunyai kaitan yang erat, maka yang menjadi rumusan masalah pada makalah ini adalah bagaimanakah sifat-sifat matriks ortogonal terkait dengan matriks ortogonal lainnya dan terkait dengan suatu transformasi linier. 
8 Adib Abdul Majid dkk

\section{Beberapa Konsep Dasar}

\subsection{Hasilkali Dalam Matriks}

Jika $\mathbf{u}=\left(u_{1}, u_{2}, \cdots, u_{n}\right)$ dan $\mathbf{v}=\left(v_{1}, v_{2}, \cdots, v_{n}\right)$ adalah vektor-vektor pada $R^{n}$, maka rumus

$$
\langle\mathbf{u}, \mathbf{v}\rangle=\mathbf{u} \cdot \mathbf{v}=u_{1} v_{1}+u_{2} v_{2}+\cdots+u_{n} v_{n}
$$

mendefinisikan $\langle\mathbf{u}, \mathbf{v}\rangle$ sebagai hasilkali dalam Euclidean pada $R^{n}$. Misalkan

$$
\mathbf{u}=\left[\begin{array}{c}
u_{1} \\
u_{2} \\
\vdots \\
u_{n}
\end{array}\right] \text { dan } \mathbf{v}=\left[\begin{array}{c}
v_{1} \\
v_{2} \\
\vdots \\
v_{n}
\end{array}\right]
$$

adalah vektor-vektor pada $R^{n}$ (yang dapat dinyatakan sebagai matriks $n \times 1$ ), dan $A$ adalah sebuah matriks $n \times n$ yang dapat dibalik. Dapat dinyatakan bahwa jika $\mathbf{u} \cdot \mathbf{v}$ adalah sebuah hasilkali dalam Euclidean pada $R^{n}$, maka rumus

$$
\langle\mathbf{u}, \mathbf{v}\rangle=A \mathbf{u} \cdot A \mathbf{v}
$$

mendefinisikan sebuah hasilkali dalam, rumus di atas disebut sebagai hasilkali dalam pada $R^{n}$ yang dihasilkan oleh $A$.

Hasilkali dalam Euclidean $\mathbf{u} \cdot \mathbf{v}$ dapat dituliskan sebagai hasilkali matriks $\mathbf{v}^{T} \mathbf{u}$, sehingga dapat dituliskan dalam bentuk alternatifnya

$$
\langle\mathbf{u}, \mathbf{v}\rangle=(A \mathbf{v})^{T} A \mathbf{u}
$$

atau secara ekuivalen,

$$
\langle\mathbf{u}, \mathbf{v}\rangle=\mathbf{v}^{T} A^{T} A \mathbf{u}
$$

\subsection{Matriks Ortogonal}

Definisi 2.1. [1] Sebuah matriks bujursangkar A yang memiliki sifat

$$
A^{-1}=A^{T}
$$

disebut sebagai matriks ortogonal.

Berdasarkan definisi di atas diperoleh bahwa

$$
\begin{aligned}
A^{-1}=A^{T} & \Leftrightarrow A A^{-1}=A A^{T} \\
& \Leftrightarrow I=A A^{T}
\end{aligned}
$$

dan

$$
\begin{aligned}
A^{-1}=A^{T} & \Leftrightarrow A^{-1} A=A^{T} A \\
& \Leftrightarrow I=A^{T} A .
\end{aligned}
$$

Oleh karena itu, $A$ adalah matriks ortogonal jika

$$
A A^{T}=A^{T} A=I .
$$




\subsection{Transformasi Linear}

Misalkan $V=\mathbb{R}^{n}$ dan $W=\mathbb{R}^{m}$, maka transformasi linier $T$ dari $\mathbb{R}^{n}$ ke $\mathbb{R}^{m}$ dituliskan sebagai berikut[1]:

(1) Untuk setiap $u$ dan $v \in \mathbb{R}^{n}$ maka $T(u+v)=T(u)+T(v)$.

(2) Untuk setiap $u \in \mathbb{R}^{n}$ dan $k \in \mathbb{R}$ maka $T(k u)=k T(u)$.

Jika $m=n$ maka transformasi $T$ disebut juga operator linier. Transformasi linier dari $\mathbb{R}^{n}$ ke $\mathbb{R}^{m}$ ini dapat dituliskan sebagai berikut [1]:

$T\left(x_{1}, x_{2}, \cdots, x_{n}\right)=\left(w_{1}, w_{2}, \cdots, w_{m}\right)$, dimana

$$
\begin{gathered}
w_{1}=a_{11} x_{1}+a_{12} x_{2}+\cdots+a_{1 n} x_{n} \\
w_{2}=a_{21} x_{1}+a_{22} x_{2}+\cdots+a_{2 n} x_{n} \\
\vdots \\
w_{m}=a_{m 1} x_{1}+a_{m 2} x_{2}+\cdots+a_{m n} x_{n} .
\end{gathered}
$$

Persamaan di atas dapat dinotasikan dalam bentuk matriks sebagai berikut:

$$
\left[\begin{array}{c}
w_{1} \\
w_{2} \\
\vdots \\
w_{m}
\end{array}\right]=\left[\begin{array}{cccc}
a_{11} & a_{12} & \cdots & a_{1 n} \\
a_{21} & a_{22} & \cdots & a_{2 n} \\
\vdots & \vdots & \ddots & \vdots \\
a_{m 1} & a_{m 2} & \cdots & a_{m n}
\end{array}\right]\left[\begin{array}{c}
x_{1} \\
x_{2} \\
\vdots \\
x_{n}
\end{array}\right]
$$

atau dapat disingkat dengan $\mathbf{w}=A \mathbf{x}$.

Matriks $A=\left[a_{i j}\right]$ disebut matriks standar untuk transformasi linier $T$, untuk $1 \leq i \leq m$ dan $1 \leq j \leq n$.

\section{Matriks Ortogonal dan Sifat-Sifatnya}

Teorema 3.1. [2] Untuk matriks $A n \times n$, pernyataan berikut ekivalen:

(1) A adalah matriks ortogonal

(2) $\|A \boldsymbol{x}\|=\|\boldsymbol{x}\|$ untuk semua $\boldsymbol{x}$ pada $R^{n}$

(3) $A \boldsymbol{x} \cdot A \boldsymbol{y}=\boldsymbol{x} \cdot \boldsymbol{y}$ untuk semua $\boldsymbol{x}$ dan $\boldsymbol{y}$ pada $R^{n}$

(4) Kolom-kolom dari matriks A membentuk sebuah basis ortonormal di $R^{n}$

(5) $A^{T} A=I_{n}$

(6) $A^{-1}=A^{T}$

Bukti. $(1) \Rightarrow(2)$. Misalkan bahwa $A$ ortogonal, sehingga $A^{T} A=I$. Ambil se- 
$10 \quad$ Adib Abdul Majid dkk

barang vektor $\mathbf{x} \in R^{n}$, perhatikan bahwa

$$
\begin{aligned}
\|A \mathbf{x}\|^{2} & =\langle A \mathbf{x}, A \mathbf{x}\rangle \\
\text { misalkan } & \mathbf{w}=A \mathbf{x} \\
= & \langle\mathbf{w}, \mathbf{w}\rangle \\
= & (A \mathbf{w})^{T}(A \mathbf{w}) \\
= & \left(\mathbf{w}^{T} A^{T}\right)(A \mathbf{w}) \\
= & \mathbf{w}^{T}\left(A^{T} A\right) \mathbf{w} \\
= & (A \mathbf{x})^{T} A \mathbf{x} \\
& =\mathbf{x}^{T} A^{T} A \mathbf{x} \\
& =\mathbf{x}^{T} I \mathbf{x} \\
& =\mathbf{x}^{T} \mathbf{x} \\
& =\langle\mathbf{x}, \mathbf{x}\rangle \\
& =\|\mathbf{x}\|^{2}
\end{aligned}
$$

jadi $\|A \mathbf{x}\|=\|\mathbf{x}\|$, untuk semua $\mathbf{x} \in R^{n}$.

$(2) \Rightarrow(3)$. Misalkan bahwa $A$ adalah matriks bujursangkar sedemikian sehingga $\|A \mathbf{x}\|=\|\mathbf{x}\|$ untuk setiap $\mathbf{x} \in R^{n}$. Akan dibuktikan $A \mathbf{x} \cdot A \mathbf{y}=\mathbf{x} \cdot \mathbf{y}$ untuk setiap $\mathbf{x}, \mathbf{y} \in R^{n}$, perhatikan bahwa

$$
\begin{aligned}
\|\mathbf{x}+\mathbf{y}\|^{2} & =\langle\mathbf{x}+\mathbf{y}, \mathbf{x}+\mathbf{y}\rangle \\
& =(\mathbf{x}+\mathbf{y})^{T}(\mathbf{x}+\mathbf{y}) \\
& =\mathbf{x}^{T} \mathbf{x}+2 \mathbf{x}^{T} \mathbf{y}+\mathbf{y}^{T} \mathbf{y} \\
& =\|\mathbf{x}\|^{2}+2 \mathbf{x}^{T} \mathbf{y}+\|\mathbf{y}\|^{2}
\end{aligned}
$$

dengan cara yang sama, diperoleh

$$
\begin{aligned}
\|A(\mathbf{x}+\mathbf{y})\|^{2} & =\|A \mathbf{x}+A \mathbf{y}\|^{2} \\
& =\langle A \mathbf{x}+A \mathbf{y}, A \mathbf{x}+A \mathbf{y}\rangle \\
& =(A \mathbf{x}+A \mathbf{y})^{T}(A \mathbf{x}+A \mathbf{y}) \\
& =\|A \mathbf{x}\|^{2}+2(A \mathbf{x})^{T} A \mathbf{y}+\|A \mathbf{y}\|^{2},
\end{aligned}
$$

karena $\|A \mathbf{x}\|=\|\mathbf{x}\|$ dan $\|A \mathbf{y}\|=\|\mathbf{y}\|$ dan $\|A(\mathbf{x}+\mathbf{y})\|=\|\mathbf{x}+\mathbf{y}\|$, berarti $(A \mathbf{x})^{T} A \mathbf{y}=\mathbf{x}^{T} \mathbf{y}$. Dengan kata lain, $A \mathbf{x} \cdot A \mathbf{y}=\mathbf{x} \cdot \mathbf{y}$.

$(3) \Rightarrow(4)$. Misalkan bahwa $A$ adalah matriks bujursangkar sedemikian sehingga $A \mathbf{x} \cdot A \mathbf{y}=\mathbf{x} \cdot \mathbf{y}$ untuk setiap $\mathbf{x}, \mathbf{y} \in R^{n}$. Misal $e_{i}$ menunjukan basis vektor standar ke- $i$ untuk $R^{n}$, dan $A_{i}$ adalah kolom ke- $i$ dari $A$. Misal

$$
A=\left[\begin{array}{cccc}
a_{11} & a_{12} & \cdots & a_{1 n} \\
a_{21} & a_{22} & \cdots & a_{2 n} \\
\vdots & \vdots & \ddots & \vdots \\
a_{n 1} & a_{n 2} & \cdots & a_{n n}
\end{array}\right]
$$


Sifat-Sifat Matriks Ortogonal dan Transformasi Ortogonal 11

dimana

$$
A_{1}=\left[\begin{array}{c}
a_{11} \\
a_{21} \\
\vdots \\
a_{n 1}
\end{array}\right], A_{2}=\left[\begin{array}{c}
a_{12} \\
a_{22} \\
\vdots \\
a_{n 2}
\end{array}\right], \cdots, A_{n}=\left[\begin{array}{c}
a_{1 n} \\
a_{2 n} \\
\vdots \\
a_{n n}
\end{array}\right]
$$

Misalkan

$$
A e_{1}=\left[\begin{array}{c}
1 \\
0 \\
\vdots \\
0
\end{array}\right], A e_{2}=\left[\begin{array}{c}
0 \\
1 \\
\vdots \\
0
\end{array}\right], \cdots \cdots, A e_{n}=\left[\begin{array}{c}
0 \\
0 \\
\vdots \\
1
\end{array}\right]
$$

maka

$$
A_{i}^{T} A_{j}=\left(A e_{i}\right)^{T} A e_{j}=A e_{i} \cdot A e_{j}=\left\{\begin{array}{l}
0 \text { if } i \neq j \\
1 \text { if } i=j
\end{array}\right.
$$

Perhatikan bahwa, misalkan $i=1$ dan $j=1$

$$
\begin{aligned}
A e_{1} \cdot A e_{1} & =\left[\begin{array}{c}
1 \\
0 \\
\vdots \\
0
\end{array}\right] \cdot\left[\begin{array}{c}
1 \\
0 \\
\vdots \\
0
\end{array}\right] \\
\left(A e_{1}\right)^{T} \cdot A e_{1} & =\left[\begin{array}{llll}
1 & 0 & \cdots & 0
\end{array}\right] \cdot\left[\begin{array}{c}
1 \\
0 \\
\vdots \\
0
\end{array}\right] \\
& =[1]
\end{aligned}
$$

misalkan $i=1$ dan $j=2$

$$
\begin{aligned}
A e_{1} \cdot A e_{2} & =\left[\begin{array}{c}
1 \\
0 \\
\vdots \\
0
\end{array}\right] \cdot\left[\begin{array}{c}
0 \\
1 \\
\vdots \\
0
\end{array}\right] \\
\left(A e_{1}\right)^{T} \cdot A e_{2} & =\left[\begin{array}{llll}
1 & 0 & \cdots & 0
\end{array}\right] \cdot\left[\begin{array}{c}
0 \\
1 \\
\vdots \\
0
\end{array}\right] \\
& =[0] .
\end{aligned}
$$


Dari kolom-kolom $A e_{1}, A e_{2}, \cdots, A e_{n}$, perhatikan bahwa,

$$
\begin{aligned}
\left\|A e_{1}\right\| & =\sqrt{1^{2}+0^{2}+\cdots+0^{2}}=1, \\
\left\|A e_{2}\right\| & =\sqrt{0^{2}+1^{2}+\cdots+0^{2}}=1, \\
& \vdots \\
\left\|A e_{n}\right\| & =\sqrt{0^{2}+0^{2}+\cdots+1^{2}}=1 .
\end{aligned}
$$

sehingga kolom dari $A$ adalah ortonormal.

$(4) \Rightarrow(5)$ Diketahui kolom dari $A$ membentuk himpunan basis ortonormal di $R^{n}$. Akan dibuktikan $A^{T} A=I_{n}$. Misalkan $A_{1}, A_{2}, \ldots, A_{n}$ kolom-kolom dari $A$ dengan

$$
A_{i}^{T} A_{j}=\left(A e_{i}\right)^{T} A e_{j}=A e_{i} \cdot A e_{j}=\left\{\begin{array}{l}
0 \text { if } i \neq j \\
1 \text { if } i=j
\end{array}\right.
$$

Perhatikan bahwa

$$
A=\left[\begin{array}{llll}
A_{1} & A_{2} & \cdots & A_{n}
\end{array}\right] \Rightarrow A^{T}=\left[\begin{array}{c}
A_{1} \\
A_{2} \\
\vdots \\
A_{n}
\end{array}\right] \Rightarrow A^{T} A=\left[\begin{array}{c}
A_{1} \\
A_{2} \\
\vdots \\
A_{n}
\end{array}\right]\left[\begin{array}{llll}
A_{1} & A_{2} \cdots & A_{n}
\end{array}\right]
$$

Sedemikian sehingga

$$
\begin{aligned}
A^{T} A= & {\left[\begin{array}{c}
A_{1} \\
A_{2} \\
\vdots \\
A_{n}
\end{array}\right]\left[\begin{array}{lll}
A_{1} & A_{2} \cdots & A_{n}
\end{array}\right] } \\
= & {\left[\begin{array}{cccc}
A_{1} A_{1} & A_{1} A_{2} & \cdots & A_{1} A_{n} \\
A_{2} A_{1} & A_{2} A_{2} & \cdots & A_{2} A_{n} \\
\vdots & \vdots & \ddots & \vdots \\
A_{n} A_{1} & A_{n} A_{2} & \cdots & A_{n} A_{n}
\end{array}\right] } \\
= & {\left[\begin{array}{cccc}
1 & 0 & \cdots & 0 \\
0 & 1 & \cdots & 0 \\
\vdots & \vdots & \ddots & \vdots \\
0 & 0 & \cdots & 1
\end{array}\right]=I . }
\end{aligned}
$$

(5) $\Rightarrow(6)$ Diketahui $A^{T} A=I$, akan dibuktikan $A^{-1}=A^{T}$. Perhatikan bahwa

$$
\begin{aligned}
A^{T} A & =I \\
A^{T} A A^{-1} & =A^{-1} \\
A^{T} & =A^{-1} .
\end{aligned}
$$

$(6) \Rightarrow(1)$ Diketahui $A^{-1}=A^{T}$, berdasarkan definisi diperoleh $A$ ortogonal. 
Teorema 3.2. [2] Misal $A$ dan $B$ adalah matriks ortogonal.

(1) AB adalah matriks ortogonal

(2) Jika $A$ adalah ortogonal, maka $\operatorname{det}(A)=1$ atau $\operatorname{det}(A)=-1$

(3) Jika $A$ adalah matriks ortogonal $n \times n$, maka $A$ invertibel dan $A^{-1}$ juga ortogonal.

Bukti. (1) Misal $A B$ adalah matriks ortogonal, maka

$$
\begin{aligned}
(A B)^{-1} & =B^{-1} A^{-1} \\
& =B^{T} A^{T} \\
& =(A B)^{T} .
\end{aligned}
$$

(2) Misal $A$ matriks ortogonal,maka

$$
\begin{aligned}
A^{T} & =A^{-1} \\
\Leftrightarrow \operatorname{det}\left(A^{T}\right) & =\operatorname{det}\left(A^{-1}\right) \\
\Leftrightarrow \operatorname{det}(A) & =\frac{1}{\operatorname{det}(A)} \\
\Leftrightarrow \operatorname{det}(A) \operatorname{det}(A) & =1 \\
\Leftrightarrow(\operatorname{det}(A))^{2} & =1 \\
\Leftrightarrow \operatorname{det}(A) & = \pm 1 .
\end{aligned}
$$

(3) Misal $A$ adalah matriks ortogonal $n \times n$. Sesuai dengan definisi matriks ortogonal, perhatikan bahwa $A^{-1}=A^{T}$, dan sesuai definisi juga bahwa jika $A$ invers, maka $A$ dapat dibalik.

\section{Transformasi Linear Ortogonal}

Transformasi $T$ dapat diwakilkan dalam bentuk matriks standar. Pada transformasi linier ortogonal juga terdapat sifat yang sama seperti pada matriks ortogonal.

Definisi 4.1. [1] Sebuah transformasi linier $T: \mathbb{R}^{n} \rightarrow \mathbb{R}^{n}$ adalah ortogonal jika untuk setiap $\vec{x} \in \mathbb{R}^{n}$

$$
\|T(\vec{x})\|=\|\vec{x}\|
$$

Teorema 4.2. [2] Jika $T: \mathbb{R}^{n} \rightarrow \mathbb{R}^{n}$ adalah ortogonal dan $\vec{v} \cdot \vec{w}=0$, maka $T(\vec{v})$. $T(\vec{w})=0$.

Bukti. Karena $T$ transformasi linier, terdapat matriks $A$ yang mewakili $T$ dan untuk setiap $v, w \in \mathbb{R}^{n}$, maka berdasarkan Teorema 3.1 berlaku $A v \cdot A w=v \cdot w$, perhatikan bahwa

$$
\begin{aligned}
T(\vec{v}) \cdot T(\vec{w}) & =A(\vec{v}) \cdot A(\vec{w}) \\
& =\vec{v} \cdot \vec{w} \\
& =0
\end{aligned}
$$


14 Adib Abdul Majid dkk

\section{Ucapan Terima kasih}

Terima kasih kepada ibu Monika Rianti Helmi, M.Si, ibu Dr. Lyra Yulianti, bapak Dr. Effendi, selaku dosen penguji, yang telah memberikan kritik dan saran dalam penulisan makalah ini.

\section{Daftar Pustaka}

[1] Anton, Howard, dan Chris Rorres. 1973. Elementary Linier Algebra; Edisi ke-8. John Wiley and Sons,Inc. New York.

[2] Kuttler, Kenneth. 2009. Elementary Linier Algebra. Textbook Equity LLC. Canada.

[3] Matthews, K.R. 1991. Elementary Linier Algebra. University of Queensland.

[4] Jacob, Bill. 1990. Linear Algebra. W.H. Freeman and Company. New York. 